\title{
RESTORATION MADE POSSIBLE WITH FORCED ORTHODONTIC ERUPTION OF ENDODONTICALLY TREATED UNRESTORABLE TOOTH: A CASE REPORT
}

\author{
Dr. Sandhya Shrestha, ${ }^{1}$ Dr. Rabindra M Shrestha, ${ }^{2}$ Dr. Marisha Dahal ${ }^{3}$ \\ ${ }^{1}$ Lecturer, Kantipur Dental College \\ ${ }^{2}$ Asst Professor, Kantipur Dental College \\ ${ }^{3}$ Lecturer, KIST Medical \& Dental College
}

Email: 0omika@hotmail.com

\section{ABSTRACT}

Post endodontic restoration in cases of grossly destructed teeth are difficult as the available tooth structure is less, margins are subgingival and of compromised prognosis because the restoration might encroach upon the periodontium affecting its status to deteriorate. Interdisciplinary approach using forced eruption or Orthodontic extrusion along with minimal surgical crown lengthening can give adequate supragingival tooth surface for proper restoration and protect its root support from getting violated. This is the case report of an unsalvageable tooth that is restored to satisfactory functional and esthetic restoration utilizing interdisciplinary approach.

\section{INTRODUCTION}

The restoration of teeth in which cervical margin impinge on the periodontal attachment continues to cause concern among restorative dentists and periodontists. ${ }^{1}$ Multidisciplinary approach is necessary to conserve the tooth with advanced caries with deep carious margins, traumatic destruction or fracture of clinical crown, iatrogenic cervical root perforation, external and internal root resorption near alveolar crest or overzealous tooth preparation and isolated infrabony defect.

These conditions are usually managed with extraction, surgical crown lengthening, surgical intra alveolar transplantation or orthodontic extrusion. ${ }^{2,3,4}$ Orthodontic extrusion or forced eruption aims to provide both sound tooth margin for ultimate restoration and to create periodontal environment (biologic width) that will be easy for patient to maintain. ${ }^{5}$ Surgical procedure alone designed to achieve this objective often compromise esthetic appearance as it widens the embrasure and entails excessive removal of supporting bone of the adjacent tooth. ${ }^{1}$

Principals of forced eruption were documented in dental literature by Angle before 1900. Heithersay In 1973 and Ingber in 1974 reported new methods of combining endodontic and orthodontic treatment to provide solution to such complicated cases. ${ }^{6}$
Orthodontic extrusion (Heithersay 1973) has been referred to as forced eruption, orthodontic eruption, vertical extrusion or assisted eruption. The technique entails orthodontic tooth movement bodily in occluso-apical vertical direction. It restores the physiological periodontal attachment and preserves alveolar bone. There are several treatment protocols for forced eruption using both fixed and removable orthodontic appliances. However, patient cooperation is a critical factor whilst using a removable appliance.

After orthodontic extrusion is completed, the tooth should be stabilized for a period of 7-14 weeks. It has been reported that with the single tooth extrusion techniques, the alveolar bone and gingiva extrude with the tooth, so gingivoplasty is advised just before the beginning of the stabilization period. Some authors have suggested a sulcular incision performed at every follow-up visit. Orthodontic extrusion requires multiple visits with excellent patient cooperation whereas surgical extrusion is a procedure which is simpler and less time-consuming. ${ }^{7}$

\section{CASE REPORT}

The present case illustrates the management of endodontically treated unrestorable tooth due to subgingival carious margin, to a successful restoration of tooth by using multidisciplinary approach. 
A 40 year old woman reported to the dental clinic of Kathmandu Model Hospital and presented with fractured palatal cusp in respect to maxillary left first premolar (24) extended subgingivally. The patient had undergone buccal ceramic facing metal crown on 25 , and silver reinforced cement restoration on 26 . The cause of the fractured palatal cusp on 24 was due to extensive carious lesion up to subgingival margin on the palatal surface (Fig. 1). The buccal plate of the enamel and dentin was intact. Pulp was involved and but asymptomatic due to chronic nature. Patient wished to restore the tooth.

Root canal treatment was performed and the canal was obturated with gutta percha by lateral condensation and was temporized with resin based Zinc oxide eugenol cement. Occlusal reduction of about $2.5 \mathrm{~mm}$ was done on buccal cusp of 24 to allow extrusion space.

Direct bond 0.018 edgewise orthodontic brackets were bonded segmentally on buccal surface of $23,24 \& 25$ (Fig 2). Initially, multi-stranded stainless steel twist flex wire was ligated to the brackets. The arch wire was progressed to 0.016 stainless steel; then to $0.016 \mathrm{X} 0.022$ stainless steel with gradual step down bends on each step in respect to 24 (Fig 3 \& 4). The arch wires were bent conforming to the segmental arch form. The patient was examined on every 15 days for a period of 8 weeks. Finally, the passive stabilizing wire was placed for retention for six more weeks.

By the accomplishment of adjunctive orthodontic treatment; supra gingival healthy tooth margin was achieved by about $2 \mathrm{~mm}$. Hence the tooth was prepared to receive cast metal onlay with dowel core up to middle third of the root. The extension of the onlay was up to cervical third of the root surface on palatal surface and up to the occlusal coverage on buccal surface (Fig 5). A hole or space was created up to the middle third of the palatal cusp of the cast restoration and the cavity was filled with acrylic resin in order to reduce the weight of the metal. The restoration was cemented with Hi-bond glass ionomer luting cement (Fig $6 \&$ 7). Postoperative IOPA radiograph after six weeks showed acceptable result.

After the retention period of six weeks; brackets were bebonded (Fig 8). Dark coloured enamel surface and metallic margin of the restoration were made esthetically acceptable by composite veneering (Fig 9); which also masked the deficient tooth structure in the cervical constriction. Patient was satisfied and a 2 year follow-up showed good condition of the tooth due to practice of oral hygiene maintenance by the patient.

\section{DISCUSSION}

Endodontic-orthodontic co-treatment becomes necessary to restore the tooth with extensive carious lesions with deep margins, traumatic destruction or fracture of clinical crown. Such teeth may not offer sound tooth structure on which to a restoration may be planned. Adjunctive orthodontic therapy will improve the existing periodontal environment by modifying the osseous topography and minimizing the need to remove the supporting bone on adjacent teeth hence reducing aesthetic compromise. This occurs when the movement of the tooth in an occlusal direction carries the connective tissue attachment in the same direction. ${ }^{8}$ The basic principal in such treatment approach is to provide adequate support to the restoration without endangering the biologic width required to maintain periodontal health necessary for the longitivity of the tooth. Animal and human studies have shown no difference in orthodontic movement between endodontically treated teeth and vital teeth. ${ }^{9}$ In the present case, the operator had no clinical difficulty in forced orthodontic eruption.

Orthodontic root extrusion techniques can offer an excellent treatment option for severely compromised clinical crown that would otherwise be non restorable except by surgical technique. The adjunctive orthodontic tooth extrusion carried out in the present case report is a simple option to save a virtually non restorable tooth which we may encounter often in our practice. The patient's desire to conserve such grossly destructed or subgingivally fractured tooth may be possible without surgical intervention provided the case is selected properly.

\section{CONCLUSION}

Orthodontic root extrusion techniques can offer an excellent treatment option for severely compromised clinical crown that would otherwise be non restorable except by surgical technique. The adjunctive orthodontic tooth extrusion carried out in the present case report is a simple option to save a virtually non restorable tooth which we may encounter often in our practice. The patient's desire to conserve such grossly destructed or subgingivally fractured tooth may be possible without surgical intervention provided the case is selected properly. 


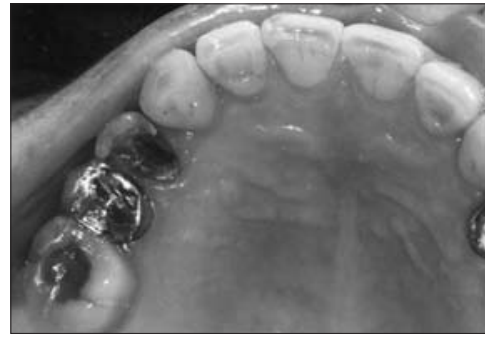

Fig 1: Extensive carious lesion on 24 (palatal view)

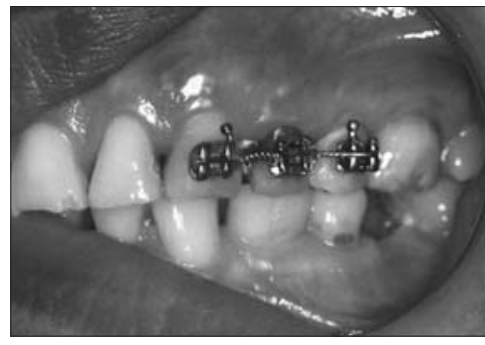

Fig 4: Rectangular stainless wire ligated on $23,24,35$ after extrusion of 24

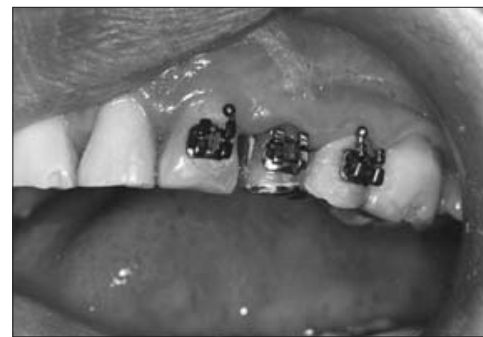

Fig 7: The restoration cemented with luting cement (buccal view)

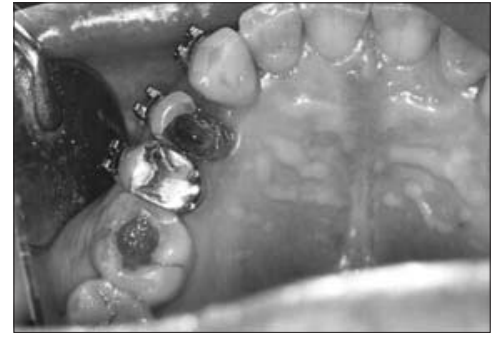

Fig 2: Orthodontic brackets bonded segmentally on 23,24 \& 25

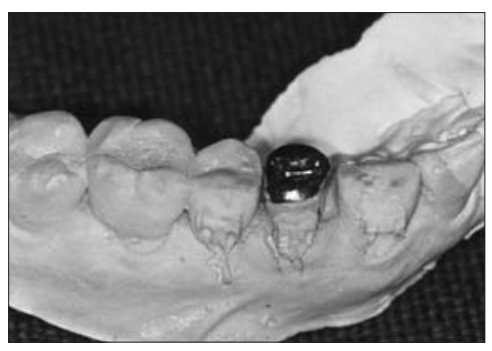

Fig 5: Cast onlay trial on dental model

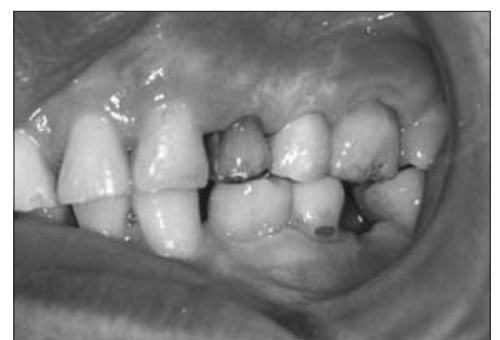

Fig 8: Brackets bebonded

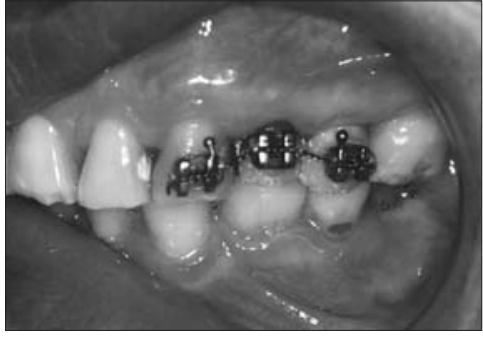

Fig 3: Multistranded twistflex wire ligated on 23, 24, 35

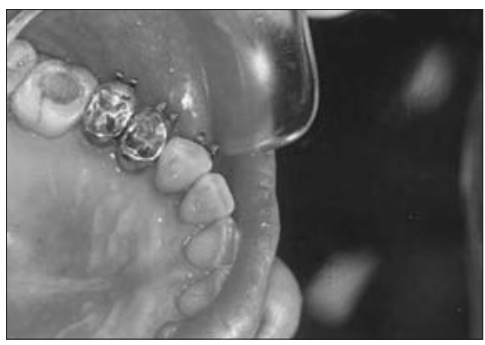

Fig 6: The restoration cemented with luting cement (palatal view)

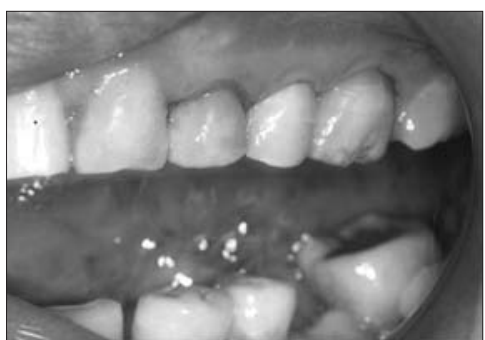

Fig 9: Esthetic Composite veneer on 24

\section{REFERENCES}

1. Scott Ross et al. Orthodontic extrusion: A multidisciplinary treatment approach. JADA 1981; 102:189-191.

2. Oesterle LJ, Wood LW: Raising the root: A look at orthodontic extrusion. J Am Dent Association 1991; 122:193-198.

3. Baker IM. Esthetic extrusion of a non restorable tooth. J Cli Orthod 1990;24:323-325.

4. Hamid Jafarzadeh, Ali Talati, Mohd Basafa \& Saeed Noorahian. Forced eruption of adjoining maxillary premolars using a removable orthodontic appliance: A case report. Journal of Oral Science 2007; 49(1):75-78.

5. RS Hamilton \& J L Gutmann: Endodontic-orthodontic relationships: A review of integrated treatment planning challenges. IEJ 1999; 32:343-360.

6. Kathuria P et al. Endodontic miscellany: Endo-ortho management of subgingival transversely fractured tooth. Endodontology 2001; Vol 13

7. V. Aggarwal, A. Logani \& N. Shah. Complicated crown fractures: Mmanagement and treatment options. International Endodontic Journal 2009; 42:740-753.

8. Weine FS. Endodontic -Orthodontic relationships. Endodontic therapy. 2004, $6^{\text {th }}$ Ed. $482-497$

9. Venkata Ramana V, Swapna M, Rajasigamani K, Gowrisankar S, SrinivasRao K. Endo-orthodontics- inside and outside the root -Interactions: tasks to be tackled. Annals and Essences of Dentistry 2010 July-Sept; 2(3): 141-8. 